\title{
COMMUNICABLE DISEASES REPORT, NSW: APRIL-MAY 2003
}

\section{TRENDS}

Summaries of case notifications through to March 2003 are shown in Figure 1, Tables 3 and 4.

\section{BLOOD-BORNE AND SEXUALLY TRANSMISSIBLE INFECTIONS}

Quarterly report: HIV notifications to end of December 2002

Tables 1 and 2 summarise recent trends in human immunodeficiency virus (HIV) disease in NSW.

\section{New HIV diagnoses}

Preliminary data indicate that notifications of new HIV diagnoses plateaued in 2002, with 350 cases reported up to the end of December (Table 1). This number compares with 348 in 2001 and 360 in 2000 . Of the 350 new diagnoses of HIV in 2002, 92 per cent were in men, 90 per cent were in people aged between 20 and 49 years, and 86 per cent lived in the Sydney metropolitan area (Table 2). Male-to-male sexual contact (with or without a history of injecting drug use) was reported as a risk factor for over two-thirds of cases, and heterosexual contact (as the only risk factor) for 15 per cent. Eight cases (two per cent) reported injecting drug use as their only risk factor.

\section{AIDS diagnoses and AIDS deaths}

Preliminary data for 2002 indicate that the number of acquired immunodeficiency syndrome (AIDS) diagnoses continued to fall in 2002 (to 66, compared with 74 in 2001 and 118 in 2000). Similarly, deaths from AIDS declined to 13 in 2002, compared with 35 in 2001 and 70 in 2000 (Table 2). Compared with people newly diagnosed with HIV infection, people reported with AIDS were more frequently men, older, resident outside of Sydney, and reported male-to-male sex as their prime risk factor for infection.

\section{GLOSSARY OF TERMS}

New HIV diagnosis refers to a person who is diagnosed for the first time with human immunodeficiency virus (HIV) infection.

Newly acquired HIV infection refers to a person with a new HIV diagnosis who tested HIV negative or reported a seroconversion illness in the 12 months before HIV diagnosis

AIDS refers to a person with HIV infection who develops one of several infections, malignancies, or other medical conditions indicating immune depression consistent with the definition of the acquired immunodeficiency syndrome (AIDS).

AIDS death refers to a person who has died of any cause after being diagnosed with AIDS.

\section{TABLE 1}

NOTIFICATION OF HIV INFECTION, AIDS AND AIDS DEATHS BY YEAR, NSW, 1981-2002

\begin{tabular}{|c|c|c|c|c|c|c|}
\hline \multirow[b]{2}{*}{ Year } & \multicolumn{2}{|c|}{ HIV } & \multicolumn{2}{|c|}{ AIDS } & \multicolumn{2}{|c|}{ AIDS deaths } \\
\hline & $N$ & $\%$ & $N$ & $\%$ & $N$ & $\%$ \\
\hline 1981 & 1 & 0.01 & 1 & 0.02 & 1 & 0.03 \\
\hline 1982 & 1 & 0.01 & 1 & 0.02 & 0 & 0.00 \\
\hline 1983 & 2 & 0.02 & 3 & 0.06 & 1 & 0.03 \\
\hline 1984 & 206 & 1.60 & 30 & 0.59 & 6 & 0.17 \\
\hline 1985 & 1005 & 7.84 & 91 & 1.77 & 46 & 1.32 \\
\hline 1986 & 1107 & 8.64 & 162 & 3.16 & 108 & 3.10 \\
\hline 1987 & 1642 & 12.81 & 251 & 4.89 & 143 & 4.10 \\
\hline 1988 & 1153 & 9.00 & 321 & 6.26 & 139 & 3.99 \\
\hline 1989 & 991 & 7.73 & 356 & 6.94 & 239 & 6.85 \\
\hline 1990 & 821 & 6.41 & 425 & 8.29 & 326 & 9.35 \\
\hline 1991 & 826 & 6.45 & 443 & 8.64 & 344 & 9.86 \\
\hline 1992 & 701 & 5.47 & 432 & 8.42 & 330 & 9.46 \\
\hline 1993 & 596 & 4.65 & 480 & 9.36 & 379 & 10.87 \\
\hline 1994 & 501 & 3.91 & 553 & 10.78 & 423 & 12.13 \\
\hline 1995 & 538 & 4.20 & 473 & 9.22 & 356 & 10.21 \\
\hline 1996 & 453 & 3.53 & 366 & 7.14 & 272 & 7.80 \\
\hline 1997 & 429 & 3.35 & 200 & 3.90 & 125 & 3.58 \\
\hline 1998 & 407 & 3.18 & 173 & 3.37 & 69 & 1.98 \\
\hline 1999 & 378 & 2.95 & 109 & 2.13 & 63 & 1.81 \\
\hline 2000 & 360 & 2.81 & 118 & 2.30 & 70 & 2.01 \\
\hline 2001 & 348 & 2.71 & 74 & 1.44 & 35 & 1.00 \\
\hline 2002 & 350 & 2.73 & 66 & 1.29 & 13 & 0.37 \\
\hline Total & 12816 & 100 & 5128 & 100 & 3488 & 100 \\
\hline
\end{tabular}




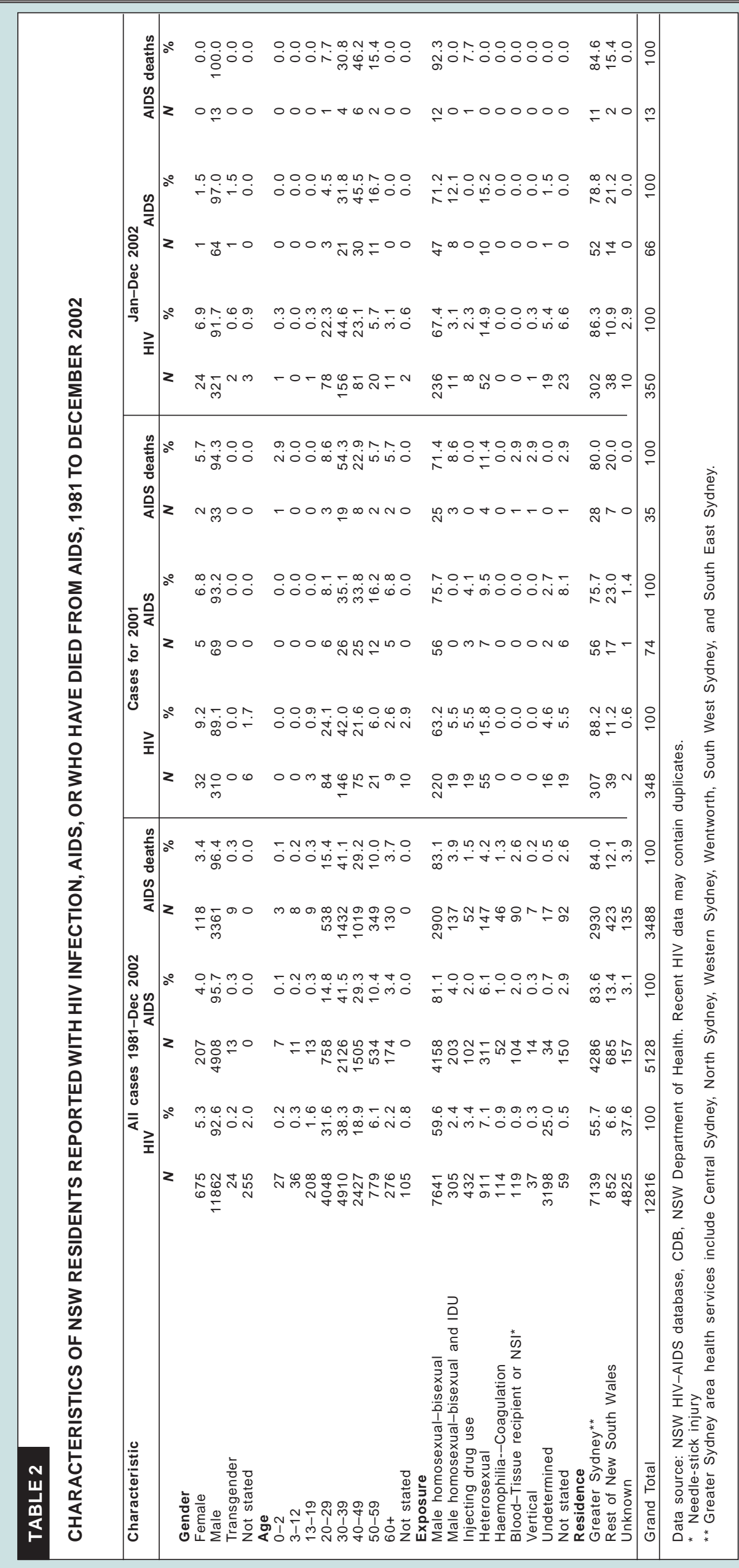


Note that recent reports of HIV diagnoses may include duplicate notifications, and that reports of HIV, AIDS, and deaths are likely to increase over time as late notifications are received.

\section{VECTOR-BORNE DISEASES}

Notifications of arboviruses remain below seasonal expectations. Most notifications received in February and March were for Barmah Forest virus infection.

\section{ZOONOSES}

\section{Case study: Brucellosis-Travellers beware}

The Western Sydney Centre for Public Health reported a case of brucellosis in February 2003. The woman had lived in the Middle East and arrived in Australia in late 2002. She presented to a GP in January 2003 with fever and pain in her left thigh. She was later admitted to hospital. Her symptoms persisted. It subsequently emerged that she had been partially treated for brucellosis before leaving the Middle East. Treatment with tetracycline was prescribed and she was re-admitted to hospital where Brucella species were detected in blood cultures.

Brucellosis is acquired through the ingestion of raw milk, or unpasteurised dairy products, or through broken skin when handling tissues, blood, body fluids, and discharges of animals infected with Brucella species. Rarely, inhalation of infected aerosols causes disease in laboratory personnel. Aerosolisation is also considered a potential vehicle for dissemination in bioterrorist attacks. Personto-person transmission of brucellosis has not been documented. In NSW, one to two cases of brucellosis have been reported annually over the last five years.

This case highlights three important messages:

1. Although rare in Australia, due principally to the effective control of the disease in the cattle and dairy industries, brucellosis remains endemic in some countries. Overseas travellers should be advised against consuming unpasteurised dairy products and handling the tissues or body fluids of animals when visiting farms. Identifying the source of infection is important, as locally-acquired cases may indicate the failure of control measures.

2. Brucellosis as a differential diagnosis is seldom considered by health care workers. It should be taken into account when symptoms including fever, sweating and chills, headache, weakness, arthralgia, depression, and weight loss are present in a patient with possible exposures in the five to 60 days before onset. Confirmation with blood culture or paired serology is essential. Laboratories must report all cases to their local public health unit. It is also helpful if clinicians notify suspected cases.

3. Language, and social and cultural factors, may act as barriers for people reporting a relevant medical history to their health care providers and health authorities. It is important that attempts to obtain information are made in a sensitive, supportive, and non-threatening environment.

\section{RESPIRATORY AND OTHER DISEASES}

Notifications of Legionnaires' disease, meningococcal disease, and invasive pneumococcal disease were in line with seasonal expectations.

\section{VACCINE-PREVENTABLE DISEASES}

\section{Measles}

No case of measles has been reported in NSW now for over seven months.

\section{Case study: Tetanus}

South Eastern Sydney Public Health Unit reported a case of tetanus in an elderly man who was admitted to hospital in February. He reported that he had injured his ankle with a rusty garden stake in January. On admission, he was noted to have a sore throat, breathing difficulties, and an ulcer on his ankle. He developed hypertonia and muscle spasms, and was transferred to an intensive care unit for ventilation. A clinical diagnosis of tetanus was made. His immunisation history was uncertain, however he reported no recent tetanus vaccinations.

Tetanus is a life-threatening disease caused by a toxin of the spore-forming bacteria Clostridium tetani. C. tetani spores survive in soil and, if they enter a wound, can grow and produce tetanus toxin. Tetanus follows an incubation period of three to 21 days, resulting in muscle rigidity, painful spasms and, among other things, respiratory failure. Tetanus is preventable through immunisation.

Immunisation against tetanus is recommended for all children at ages two, four, and six months, with boosters at 18 months and four years of age. Further boosters are recommended at 15-19 years and 50 years of age. Unimmunised adults should receive a primary course of three doses given at least a month apart, and then two boosters at 10-year intervals. Management of tetanusprone wounds depends on a history of vaccination and the type of wounds sustained, and may involve a dose of tetanus toxoid, and tetanus immunoglobulin (see the Australian Immunisation Handbook for details).

\section{ENTERIC DISEASES}

\section{Salmonellosis \\ February}

The NSW Department of Health received 275 notifications of salmonellosis in February, of which 48 per cent were due to the Salmonella enterica serovar Typhimurium ( $S$. Typhimurium)(STM). The most commonly notified phage types were STM 170 and STM 197. Public health units in areas where cases have been notified have been asked to 
interview at least two cases of both of these infections to help identify any common risk factors. Other serovars with notable increases in February include $S$. chester, S. infantis, and S. virchow. Northern Sydney Public Health Unit investigated five cases of $S$. virchow but no common links were identified.

March

In March, there was an increase in notification of a number of Salmonella serovars. There were 18 cases of S.chester reported compared with no cases in 2002 and five cases in 2001. STM 170 and 197 continued to be reported frequently. Interviews with cases were conducted but these did not reveal either a likely source of infection or links between the cases. No source for the increase in $S$. virchow phage type 19 in February was determined.

\section{Listeriosis}

Three cases of listeriosis were notified in February and two cases in March. Of these, three had underlying immunocompromising conditions. No common links have been found among the cases.

\section{Haemolytic Uraemic Syndrome}

One case of Haemolytic Uraemic Syndrome (HUS) was reported in March in a four-year-old girl.

\section{Outbreaks}

Eleven of twenty-four people who attended a private party in the Illawarra Area became ill with diarrhoea and vomiting in February. The Salmonella phage type STM 135a was isolated from three stool samples and Salmonella species were isolated from a sample of left over food. Interviews with party-goers who became ill could not determine the particular food responsible for the outbreak.

Three other outbreaks of gastroenteritis were reported in February. Two of these occurred in aged care facilities, one of which was confirmed to be caused by a Norovirus (the new name for Norwalk virus).

The Hunter Public Health Unit recently investigated an outbreak of gastroenteritis among students and staff following a secondary school camp. Of the 96 people on the camp, 19 reported symptoms that included fever, diarrhoea, vomiting, and abdominal cramping. Two cases had bloody bowel motions. Campylobacter was isolated from three of the four stool samples collected. The most likely source of infection was cooked take-away chickens. These chickens had been held at a temperature between 5-60 degrees Celcius for over four hours before being served and leftovers were also consumed the following day. Poor quality drinking water was another possible source of infection. Samples of tank water and untreated dam water available through local taps at the campsite both contained raised coliform counts and E. coli but no other bacterial pathogens were isolated.

\section{SEVERE ACUTE RESPIRATORY SYNDROME (SARS)}

\section{Summary of SARS investigations}

In the week ending 25 April 2003, there were two people identified in NSW who had fever and respiratory symptoms within 10 days of being in a SARS-affected area. Both recovered well and were released from hospital. In total, 33 patients have been investigated for SARS in NSW since 17 March 2003, including eight people with pneumonia. None are thought to have SARS.

\section{Background}

An epidemic of atypical pneumonia was first noted in southern China in November 2002. Subsequent transmission to travellers and their contacts led the World Health Organization to issue a global alert on 15 March 2003. The disease, which included fever and respiratory symptoms, was named Severe Acute Respiratory Syndrome or SARS.

The case definition for suspected SARS included a person who visited an affected area, or who had close contact with a SARS case in the previous 10 days, who develop fever $\left(>38^{\circ} \mathrm{C}\right)$ and cough, shortness of breath, or difficulty breathing. Probable cases have a chest X-ray indicating pneumonia or respiratory distress syndrome (RDS) in the absence of another cause. However, because no causative pathogen is identified in 40 per cent of patients with community-acquired pneumonia in normal circumstances, many patients fitting this definition may well have a disease other than SARS.

A new coronavirus has now been identified as the pathogen that causes SARS. Until specific diagnostic tests for the new coronavirus are validated, definitive diagnosis of SARS remains difficult.

\section{Methods}

Active surveillance for SARS in NSW began on 17 March 2003. Hospitals and general practitioners were alerted to report possible cases to their public health unit, and to use SARS infection control precautions. The current history of all patients under investigation are reviewed by a national expert committee to determine the probability of SARS in each patient.

\section{Findings}

As of the 20 April, the Communicable Diseases Branch of the NSW Department of Health has been notified of 33 people who have been investigated for possible SARS. Four people had onset of fever in February, 13 in March, and 16 in April.

Of these 33 patients, 20 (61 per cent) were male and their ages ranged from six months to 74 years $($ median $=43$ years). Five patients (16 per cent) were aged $0-5$ years, two patients (six per cent) were aged 6-19 years, nine patients (27 per cent) were aged $20-39,11$ patients (33 
per cent) were aged $40-59$, and six patients (18 per cent) were older than 60 years.

Twenty-one patients ( 64 per cent) resided in metropolitan Sydney and eight (24 per cent) in other parts of NSW. Four patients (12 per cent) were overseas visitors.

\section{Clinical features}

All 33 patients reported fever and cough. Fifteen patients (47 per cent) also reported shortness of breath, and 12 patients (38 per cent) reported difficulty breathing.

Twenty-five patients (76 per cent) had fever and cough but either had no chest X-ray or their X-ray showed no evidence of pneumonia. An alternative diagnosis was subsequently confirmed for seven of these cases (influenza A [1], parainfluenza [2], mycoplasma [1], streptococcus pneumoniae [1], and typhoid [1]). These patients are thought unlikely to have SARS, even in the absence of an alternative diagnosis.

Of the eight patients ( 24 per cent) with pneumonia confirmed by chest X-ray, one was subsequently diagnosed with influenza $A$, one was diagnosed with mycoplasma, one was diagnosed with streptococcus pneumoniae, two had X-rays inconsistent with SARS, two responded rapidly to antibiotics, and one had only limited exposure to a SARS affected area (in transit). One person remains under investigation for possible SARS.

Eighteen patients (55 per cent) were hospitalised. None has been admitted to intensive care and none have died.

\section{Exposure}

All 33 patients reported being in an affected country, including 19 (58 per cent) in Hong Kong, eight (24 per cent) in Singapore, three (nine per cent) in China, two (six per cent) in Hanoi, Vietnam, and one (three per cent) in Toronto, Canada. Several people visited more than one affected area.

For eight patients (24 per cent), their only exposure was time spent in transit at airports (range: 1-7 hours). Of the remaining 25 people who were travellers, the mean length of stay in an affected country was nine days, (median five days; range: one to 58 days). The earliest travel to an affected area was identified as 2 February 2003. Three patients were residents of affected areas.

No patient reported close contact with a person known to have SARS. No health care worker caring for patients being investigated for SARS have been notified to NSW Health.

\section{Airport screening}

No cases of SARS have been identified from surveillance of inbound passengers at Sydney International Airport. Medical staff at the airport review between one and eight travellers per day for SARS.

For more information, access the NSW Department of Health website at:

www.health.nsw.gov.au/public-health/alerts/sars/ index.html $\mathrm{x}$

\section{NSW PUBLIC HEALTH BULLETIN}

The NSW Public Health Bulletin is a publication of the NSW Department of Health.

The editor is Dr Lynne Madden, Manager, Public Health Training and Development Branch.

Dr Michael Giffin is the managing editor.

The Bulletin aims to provide its readers with population health data and information to support effective public health action.

The Bulletin is indexed by MEDLINE and Index Medicus.

\section{Submission of articles}

The preferred length of Bulletin articles is 1500 words. Tables and figures may be additional to that. News, comments, and other reports should be 500-600 words.

All manuscripts should contain a short introductory abstract that reflects the structure of the manuscript. References should be set out in the Vancouver style.

Send submitted manuscripts on paper and in electronic form, either on disc (Word for Windows is preferred), or by email.

The manuscript must be accompanied by a letter signed by all authors.

Full instructions for authors are available on request from the managing editor.

\section{Editorial correspondence}

Please address all correspondence and potential contributions to The Managing Editor, NSW Public Health Bulletin, Locked Mail Bag 961, North Sydney, NSW 2059, Australia or by email to phbulletin@doh.health.nsw.gov.au.

Tel: 6129391 9241, Fax: 61293919232.

\section{Distribution}

To obtain copies of the NSW Public Health Bulletin please contact your local public health unit or by telephone at

61293919942.

A new subscribers-change of address form is printed in most issues of the Bulletin. There is also an online subscription form available at the Bulletin's website.

The Bulletin can be accessed in both PDF and HTML formats from www.health.nsw.gov.au/ public-health/phb/phb.html.

All back issues are downloadable from the website. Back issues of the printed version can be obtained from:

Public Health Training and Development Branch

NSW Department of Health

Locked Mail Bag 961

North Sydney, NSW 2059, Australia.

Copyright (c) 2003 NSW Department of Health

\section{NSWERHEALTH}


FIGURE 1

REPORTS OF SELECTED COMMUNICABLE DISEASES, NSW, JANUARY 1998 TO MARCH 2003, BY MONTH OF ONSET

Preliminary data: case counts in recent months may increase because of reporting delays.

Laboratory-confirmed cases only, except for measles, meningococcal disease and pertussis $\mathrm{BFV}=$ Barmah Forest virus infections, RRV $=$ Ross River virus infections

$\mathrm{LI}=$ Legionella longbeachae infections, $\mathrm{L} p=\mathrm{L}$. pneumophila infections

Gp $C$ and $G p B=$ disease due to serogroup $C$ and serogroup $B$ infection,

other/unk $=$ other or unknown serogroups

\section{Cases}
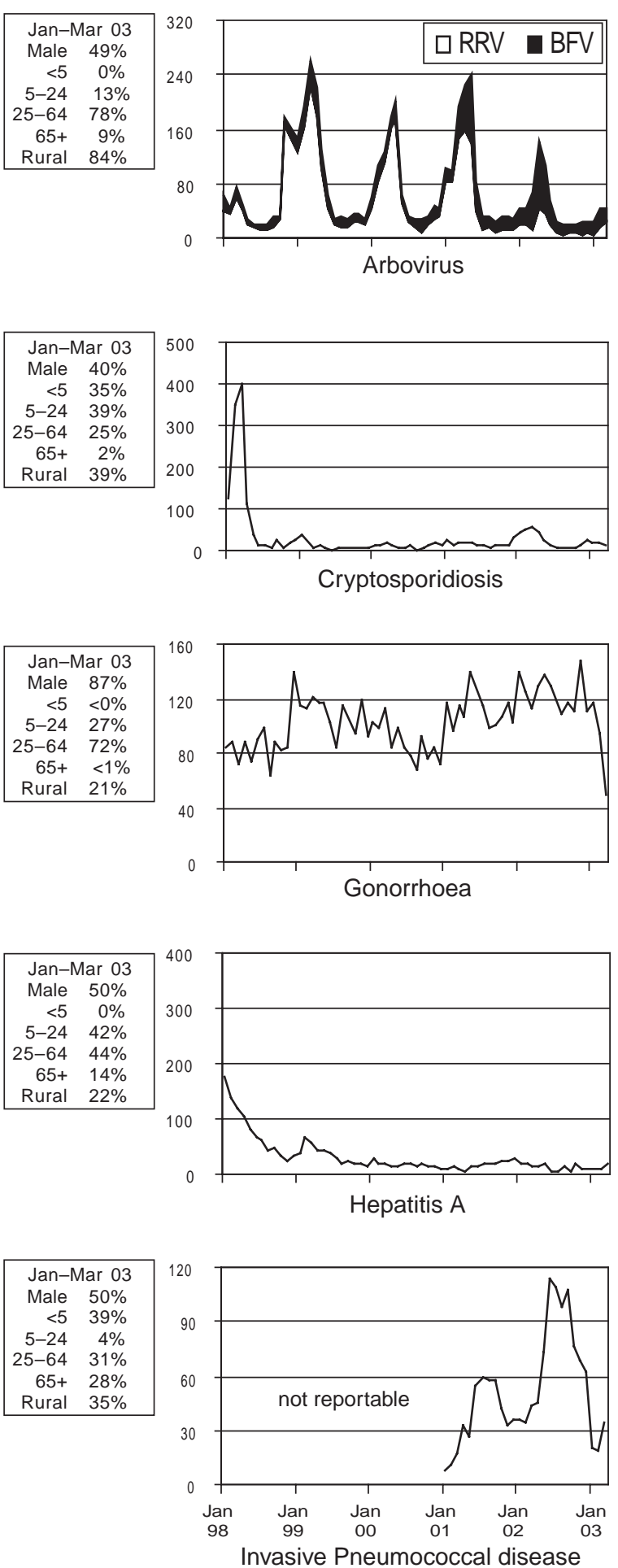

Month of onset

\section{Cases}

\begin{tabular}{|rc|}
\hline \multicolumn{2}{|c|}{ NSW population } \\
Male & $50 \%$ \\
$<5$ & $7 \%$ \\
$5-24$ & $28 \%$ \\
$25-64$ & $52 \%$ \\
$65+$ & $13 \%$ \\
Rural $^{*}$ & $42 \%$ \\
\hline
\end{tabular}
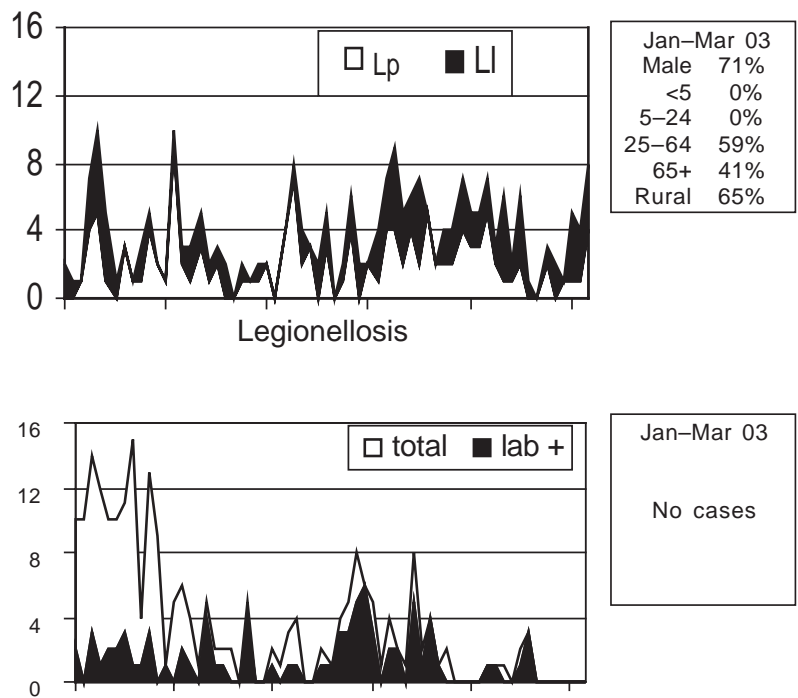

Jan-Mar 03

No cases

Measles

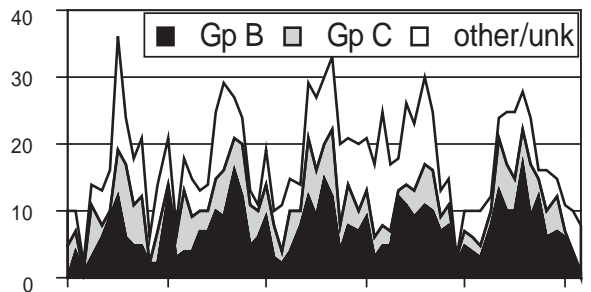

Meningococcal disease
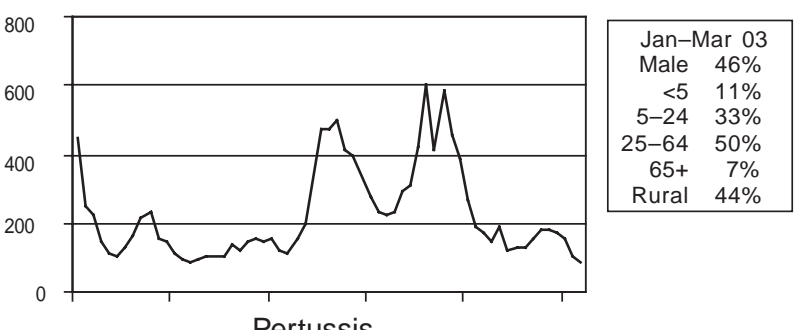

Pertussis

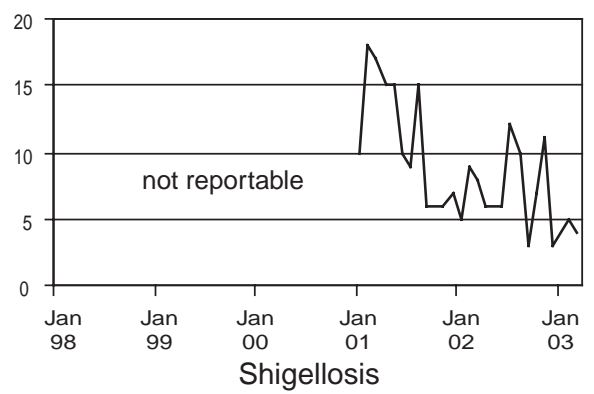

Month of onset

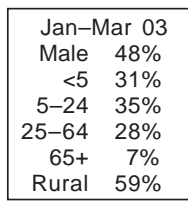

Jan-Mar 03 Male $54 \%$

$<5 \quad 0 \%$

$5-24 \quad 39 \%$

$65+\quad 8 \%$

Rural $23 \%$ 


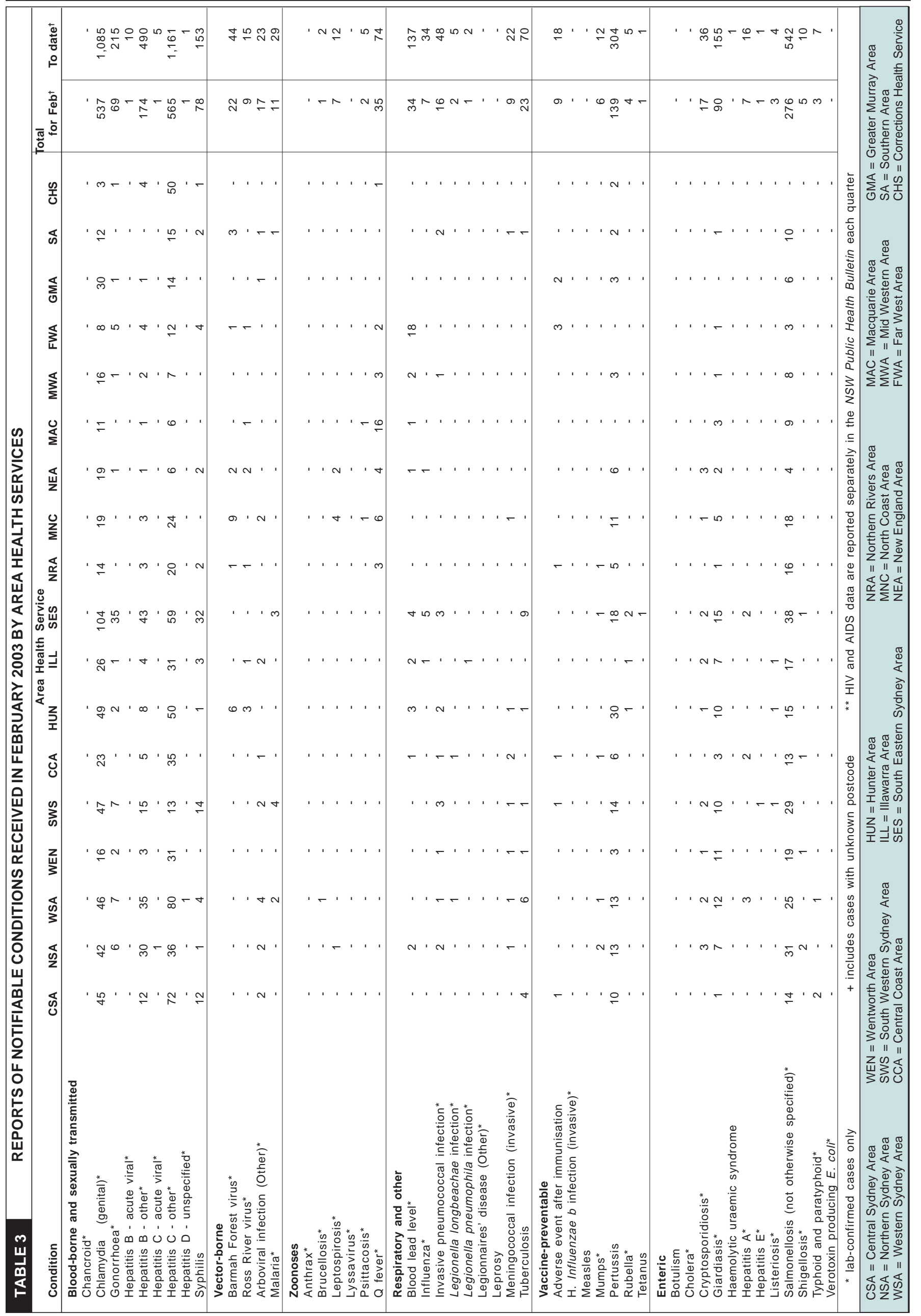




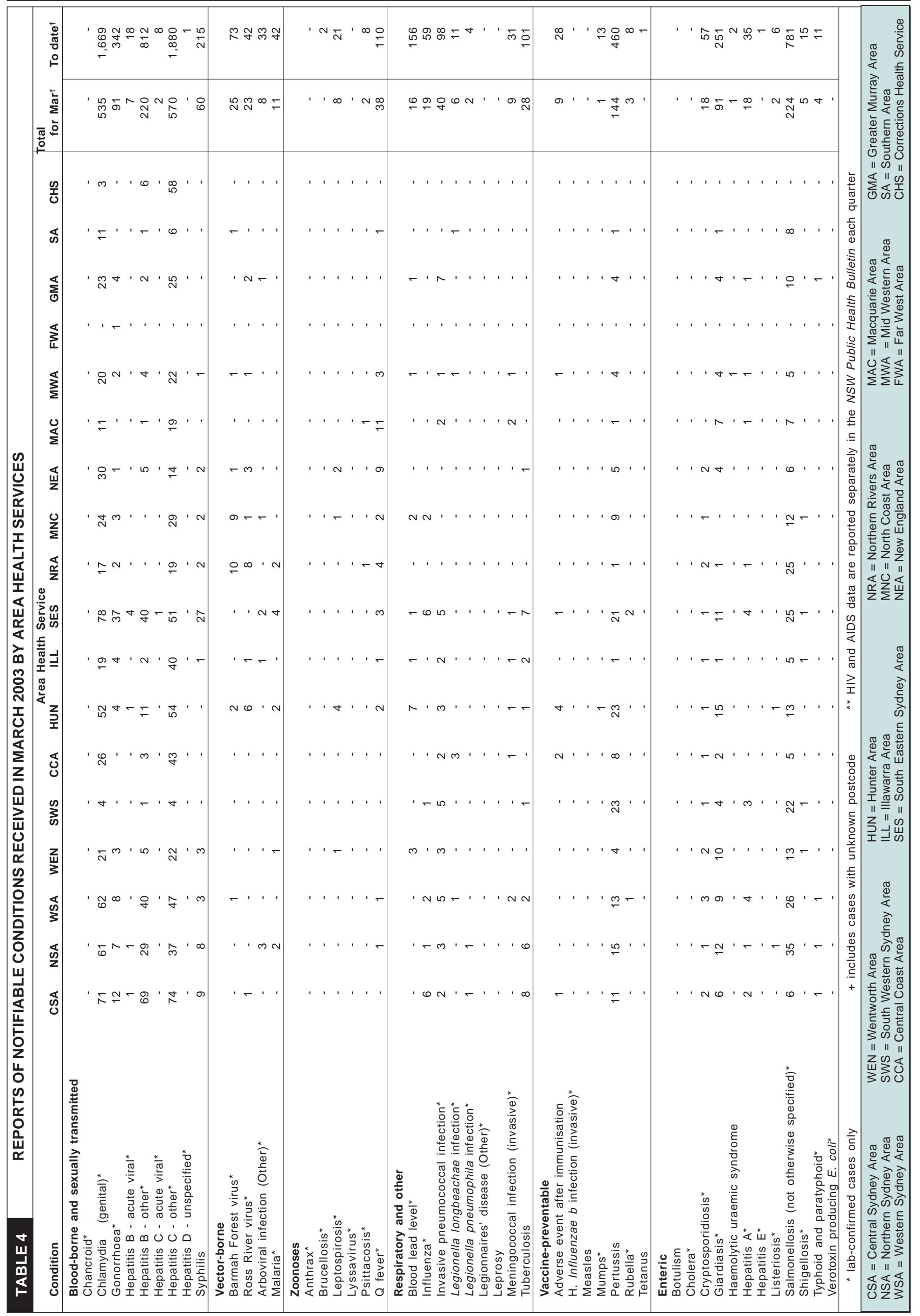

